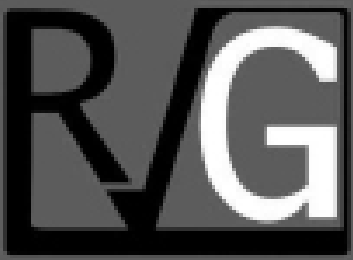

Año 24 No. 88

Octubre - Diciembre 2019

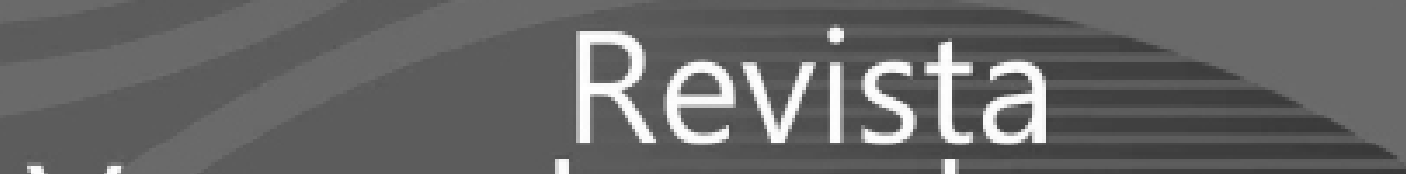

Venezolana de

verencla

UNIVERSIDAD DEL ZULIA (LUZ)

Facultad de Ciencias Económicas y Sociales

Centro de Estudios de la Empresa 


\title{
Gestión de Innovación tecnológica y globalización como factores impulsadores de la calidad de servicio y competitividad
}

\author{
Flores Ccanto, Florencio ${ }^{1}$ \\ Ramos Vera, Rosario Pilar ${ }^{2}$ \\ Ramos Vera, Fernando ${ }^{3}$ \\ Ramos Vera, Ana María ${ }^{4}$
}

\section{Resumen}

El objetivo principal de esta investigación es describir la gestión de innovación tecnológica que, junto a la globalización, se ha convertido en un factor impulsor de la calidad del servicio y la competitividad organizacional. La gestión de la innovación tecnológica implica el uso de la tecnología para el aprovechamiento de los recursos organizacionales en beneficio de los clientes razón por la cual su estudio permite delinear estrategias y acciones ajustadas a cada organización. Bajo esa perspectiva, se desarrolló está investigación de tipo documental basada en la revisión de fuentes bibliográficas y documentación de diferentes autores que abordan la temática, entre estos destacan, Betz (2011), Capdevielle (2000), Shumperter (1934), Freeman (1982), Rotwell (1986), entre otros. De lo investigado se puede concluir que la gestión de innovación tecnológica tiene

Recibido: 20-03-19 Aceptado: 18-06-19

1 Doctor en ciencias de la educación, Máster en computación y Licenciado en Educación. Docente Principal Universidad Nacional de Educación Enrique Guzmán y Valle, Perú. Email: fflores@une.edu.pe; ORCID: 0000-0001-5600-9854

2 Magister en Educación con Mención en Docencia y Gestión Educativa, Cirujano Dentista, Licenciado en Educación Secundaria Matemática, segunda especialidad investigación y didáctica en el área de Matemática, nivel de educación secundaria de educación básica regular, Docente de la Universidad Norbert Wiener y doctorando en educación Universidad Privada César Vallejo, Perú. Email: rosarioramosvera@gmail.com ORCID: 0000-0002-0712-524X

3 Magister en Educación, mención en docencia y gestión educativa, Licenciada en educación primaria, segunda especialidad en didáctica de la educación primaria y doctorando en educación. Universidad César Vallejo, Perú. Email: fer.canteno.2018@gmail.com ORCID: 0000-0001-7083-253X

4 Maestra en Educación con mención en docencia y gestión educativa, segunda especialidad en didáctica de la educación primaria y doctorando en educación Universidad César Vallejo, Perú. Email : anamariaramosvera@ gmail.com ORCID: 0000-0001-6978-3689 
Gestión de Innovación tecnológica y globalización como factores impulsadores de la calidad de servicio y competitividad

Ana María, Ramos Vera

varias aristas y diferentes puntos de vista. Desde el enfoque económico se defienden las bondades de sus avances y su impacto en el desarrollo empresarial; mientras, los más humanistas cuestionan el uso de las tecnologías por desplazar la mano de obra calificada dejando a un lado la fuerza laboral y el detrimento de las condiciones sociales de los trabajadores. Sin lugar a dudas, la globalización ha puesto al alcance de las organizaciones los resultados de las innovaciones tecnológicas las cuales, a su vez, impactan la producción de los bienes y servicios, en general, haciendo más exigente los niveles de calidad y competitividad organizacional en beneficio de los clientes.

Palabras clave: gestión tecnológica; globalización; competitividad organizacional; innovación tecnológica; calidad de servicio.

\title{
Gestión de Innovación tecnológica y globalización como factores impulsadores de la calidad de servicio y competitividad
}

\begin{abstract}
The main objective of this research is to describe the management of technological innovation that, together with globalization, has become a driving factor in service quality and organizational competitiveness. The management of technological innovation implies the use of technology for the use of organizational resources for the benefit of customers, which is why their study allows us to delineate strategies and actions tailored to each organization. From this perspective, this documentary research was developed based on the review of bibliographic sources and documentation of different authors that address the subject, among them, Betz (2011), Capdevielle (2000), Shumperter (1934), Freeman (1982 ), Rotwell (1986), among others. From what has been investigated, it can be concluded that the management of technological innovation has several edges and different points of view. From the economic approach, the benefits of its advances and its impact on business development are defended; Meanwhile, the most humanists question the use of technologies to displace skilled labor, leaving aside the labor force and the detriment of the social conditions of the workers. Without a doubt, globalization has made the results of technological innovations available to organizations, which, in turn, impact the production of goods and services, in general, making quality levels and organizational competitiveness more demanding. customer benefit.
\end{abstract}

Key Words: Technology management; globalization; organizational competitiveness; technological innovation; quality of service 


\section{Introducción}

Iniciando el siglo XXI, como consecuencia de la globalización, las empresas con mayores ganancias en el mercado han requerido la aplicación de novedosas técnicas para que sus operaciones funcionen en armonía con los nuevos tiempo, cónsonas con el denominado ahorro energético, con sistemas de producción más eficientes en pro de la disminución del impacto al medio ambiente, más preocupadas y ocupadas en los públicos organizacionales, principalmente, en los trabajadores y clientes.

De este modo, para abordar la innovación tecnológica en las organizaciones se requiere de una gestión que planifique, coordine y controle los elementos necesarios para cumplir con los programa y objetivos plateados, haciendo uso de esta innovación en todos las áreas y procesos medulares, principalmente; y en los procesos de apoyo, en la medida de las posibilidades, para garantizar una mayor eficiencia y efectividad. Lo anterior, debe estar unido a la formación en el área tecnológica de todos los usuarios, en especial de los gerentes, responsables de gestionar el buen uso de los recursos organizacionales. Escorsa y Valls (2003) señalan como ejemplo la aplicación de la estrategia tecnológica en Japón, donde casi $90 \%$ de los puestos de la alta gerencia están ocupados por ingenieros que se dedican a trabajos de gestión.

Sin lugar a dudas, la innovación tecnológica tiene como propósito principal impactar en la percepción final de los clientes, creando niveles de satisfacción y de calidad por encima del estándar y logrando el reconocimiento y posicionamiento esperado con base en la diferenciación. Para ello, las entradas (insumos, materias primas, entre otros), procesos (producción, gestión de recursos, entre otros) y salidas (bienes y servicios) deben estar alineados y responder a una gestión tecnológica eficiente.

Consecuentemente, la ausencia de políticas para la innovación tecnológica puede fragmentar el poder de decisión de las empresas, debido a que los planes o prácticas, indefinidos o ambiguos, impiden la elección de directrices claras para alcanzar los objetivos y por consiguiente conlleva a resultados deficientes, inversión de mayor tiempo en el análisis de ideas, toma de decisiones con una mentalidad a corto plazo, poca efectividad en la ejecución de proyectos, entre otras consecuencias resultado de una mala gestión (Fernández, 2005).

Por otro lado, la gestión de innovación tecnológica requiere identificar el mercado objetivo para desarrollar tecnología acorde a las necesidades; la falta de reconocimiento de las necesidades del cliente y el seguimiento discontinuo de la actuación de estos frente a los bienes o servicios prestados, genera confusión y poca confianza, inclinándose los clientes hacia los procesos y/o productos de la competencia, bien sea, por su calidad, precio, disponibilidad, o cualquier otra razón de mercado.

En este sentido, para Betz (2011) una de las situaciones más influyentes en el mercadeo de bienes y servicios en empresas, es el logro de la búsqueda de clientes potenciales de acuerdo con las especificaciones del producto. Cuando no existe la suficiente divulgación de las características y precios para lograr persuadir a los clientes a comprar un determinado producto y servicio, con mucha dificultad se logra sobrepasar las 
expectativas del consumidor. El gerente que logre ver nuevas oportunidades en la comercialización fomentará, no solo nuevos mercados, sino que liderará una nueva competencia, lo que denomina Betz (2011) una tecnología imperativa.

Es en este punto donde entra en juego la globalización como factor impulsor de la innovación tecnológica, tomando en cuenta el mercado global, las estrategias adoptadas a nivel internacional que han incidido considerablemente en el avance de la innovación, y por lo tanto en el posicionamiento de productos a escala mundial generando estándares de calidad más altos y promoviendo una mayor competitividad en las organizaciones. En este sentido, la presente investigación busca documentar como la gestión de la innovación tecnológica de la mano de la globalización impacta en la calidad del servicio y por ende mejora la competitividad organizacional, valiéndose para ello, de la investigación documental y el análisis de las propuestas teóricas revisadas.

\section{Evolución de la gestión de innovación tecnológica en las organizaciones}

Desde finales del siglo XVI grandes pensadores, filósofos y economistas ya habían considerado el tema del cambio tecnológico en los aspectos sociales y económicos, a pesar que no ha estado presente con la misma atención en el devenir de los años, de hecho se desarrollaron diversos enfoques que de alguna u otra forma han ido completando el concepto de innovación tecnológica y su influencia en el diseño de las acciones públicas, inclusive.

El filósofo y economista Adam
Smith, durante los últimos años del siglo $\mathrm{XVII}$, reconoce que la invención de la maquinaria dentro de la división del trabajo ha permitido la mayor destreza del operario, el ahorro del tiempo que se emplea en ejecutar una actividad de otra de distinta especie y la introducción de máquinas especializadas, lo que permite la realización de lo que hoy se conoce como mejora tecnológica (Smith, 1996). Otro aporte de interés se genera con lo establecido por Malthus (1973) en donde la invención de las máquinas permite el ahorro del trabajo y a su vez facilita la caída de los precios y una extensión de la demanda en la mercancía. Adicionalmente se le suma las ideas impuestas por Ricardo (1973)quien estableció que las mejoras en los medios de producción aumentan la producción neta, trayendo como consecuencia un mejor estándar de vida para todas las clases.

Similarmente, Mill en 1973, defiende la posición de las mejoras de la productividad la cual incluye la invención en herramientas y maquinarias, también reflexiona sobre el papel de las clases trabajadoras las cuales podían padecer, no solo los efectos de la inversión de capital en maquinaria, sino, también, el desplazamiento de la mano de obra. Sin embargo, tales teorías neoclásicas solo defienden la posición del equilibrio en función de la producción para los cambios tecnológicos; la visión de Marx en el siglo XIX muestra su aporte en la maquinaria y la gran industria donde admite la necesidad del capitalista en acumular capital para mantener sus ganancias y el ahorro de la fuerza de trabajo mediante incrementos salariales (Marx, 1978).

Otra visión más amplia es la de las teorías evolucionistas que van $r$ más allá del equilibrio de la producción, sino 
los motivos del por qué el mundo tan cambiante de hoy en día se enrumba a nuevas estructuras sociales, económicas y políticas. Ahora bien, ¿cuál es la teoría más cercana a la innovación tecnológica de las organizaciones actuales?, es una pregunta que puede ser respondida recorriendo los aportes de filósofos y economistas que desde el siglo XVII hasta la llegada de la época actual en el siglo XXI han analizado los indicadores económicos, sociales, políticos que definen la innovación como eje impulsor del desarrollo de la industria actual.

Así se tiene el caso del famoso sociólogo Veblen en su proyecto en 1965 denominado: "Theengineers and the Price system", traducido al español como: "Los ingenieros y el sistema de precios" quien fue precursor de la teoría Schumpeteriana, observó que en la primera guerra mundial se requirió de mayor innovación industrial y predijo la necesidad de hacer cambios en las estructuras gerenciales, de igual manera también afirmó que los bienes o servicios producidos dependían más de los gerentes tecnócratas y de los innovadores de capital que de los gerentes financieros.

No obstante, aún los conceptos que le preceden a Veblen son considerados insuficientes para la definición de innovación, para lo cual ninguno de los precursores de Schumpeter ha sido tan influyente como él mismo, en este sentido, Schumpeter en el siglo XVIII introduce una nueva concepción en la economía; pensaba que las preferencias de los clientes eran influenciadas y no surgían de forma espontánea, por lo tanto. los clientes no podían ser una causa del cambio económico. En su teoría del desarrollo económico, lo describe como un proceso histórico de cambios estructurales manejados por la innovación la cual fue clasificada por cuatro etapas que se mencionan a continuación (Shumperter, 1934):

a. El lanzamiento de un nuevo producto o de nuevas especies de un producto conocido.

b. La aplicación de nuevos métodos de producción o de ventas de un producto (no probado aún en la industria)

c. La apertura de un nuevo mercado (el mercado a través del cual una rama de la industria aún no ha sido representada)

d. La adquisición de nuevas fuentes de materia prima o bienes semi - elaborados.

e. Una nueva estructura en la industria tal como la creación o la destrucción de un monopolio.

Schumpeter (1934), hizo un gran énfasis en el hecho de que para obtener mayor ganancia era necesario, sin duda, innovar, enfatizando, que la innovación era el motor esencial para la competitividad y la dinámica económica, en sus escritos resaltó la innovación como el centro del cambio económico, introdujo el concepto de destrucción creativa, pues la innovación es un proceso de mutación industrial que revoluciona incesantemente la estructura económica, destruyendo incesantemente la anterior e incesantemente creando una nueva (Shumpeter, 1934).

Habría que decir también que, Schumperter introduce otro término del cual no se había hablado mucho acerca del papel del empresario en la innovación de las organizaciones, mencionando en sus trabajos dos visiones: La primera enfocada a destacar una definición funcional que solo se le confiere a aquel que reforma o revoluciona la patente de la producción mediante la explotación de la invención o de forma más general, la 
posibilidad tecnológica poco usual para producir un nuevo bien o transformando un producto viejo a una nueva forma, creando una nueva fuente de materiales así como también una nueva salida de productos, reorganizando la industria. Asimismo, el empresario no necesariamente recibe las ganancias, pues el que las recibe es el capitalista o el dueño de la compañía. De hecho, se menciona tres motivos por los que se define a un empresario, primero el deseo de crear su propio reino o dominio de independencia, el deseo de ganar sobre otros y el juego de la creación mediante el ingenio de diseñar nuevos productos o sistemas (Schumpeter, 1934).

La segunda teoría de Schumpeter acerca del espíritu emprendedor, donde el empresario no necesariamente tiene que ser una persona, sino más bien que este papel puede ser perfectamente asumido por los estados de una nación. Además, consideró que entre los tres ciclos de búsqueda: el teorético, estadístico y el histórico, es el último más valorable que el resto (Schumpeter, 1934).

Por lo tanto, la idea principal de la generación de la innovación en el progreso dinámico y tecnológico no depende de personalidades individualistas, sino que envuelve la cooperación de diferentes autores y de allí la evolución del concepto de empresario que tuvo Schumpeter en su continuo estudio. Las investigaciones de Schumpeter en aquel entonces han sido las más apropiadas para describir que actualmente el capitalismo está experimentando una crisis seria y ha perdido su fuerza impulsora por la crisis económica existente, por lo que se busca las mejores actuaciones en el mundo tan cambiante de hoy en día.

Luego de Schumpeter (1934), como principal referente para innovación, desataca Rogers (1962), proponiendo una definición de innovación más restrictiva, pudiendo ser combinado con un adjetivo tal como tecnológica $u$ organizacional. La mayor parte de su trabajo acerca de la innovación estuvo enfocado en la innovación tecnológica, estableciendo el concepto de la difusión de innovación como el proceso mediante el cual se logra la difusión de una idea que va desde su fuente de invención o inicio hasta sus últimos usuarios o adoptantes La importancia de la difusión radica en la interacción humana mediante la cual una persona le comunica a otra una nueva idea, para el autor, este proceso se lleva a cabo a través de cuatro etapas: la innovación, la comunicación de un individuo a otro, el sistema social y el tiempo(Rogers, 1962).

Ahora bien, siguiendo el mismo orden de ideas, Freeman (1982) en su publicación intitulada: "La economía de la innovación industrial", consideró que la innovación debe entenderse en un sentido más amplio como un proceso interactivo de las organizaciones donde, además de adquirir conocimientos acerca del diseño, el desarrollo, la producción y la comercialización, también se obtiene un enriquecimiento de sus relaciones con fuentes externas, las cuales involucran proveedores, consumidores, instituciones, tales como, universidades, centros de investigación o incluso hasta empresas de la competencia. El establecimiento de este tipo de relaciones o la conformación de acuerdos de cooperación entre las organizaciones permitirá ahondar más en la innovación tecnológica.

Inclusive, además de la relación de los factores externos de una organización para el desenvolvimiento de la innovación tecnológica, el autor 
destaca, la relevancia de los factores internos como motores de aprendizaje, representado en la acumulación interna de conocimientos a través de procesos de formación de personal o con conocimiento preconcebido aunque, si bien es cierto, afirmó que era necesario profundizar su estudio para ser medido correctamente (Freeman, 1982)

Ahora bien, para el caso específico de Rothwell (1986), la innovación es un proceso que además de tomar en cuenta el desarrollo y la investigación, así como, reconocer las necesidades del mercado, estas vertientes deben conducir a una retroalimentación del bien o servicio ofrecido ya que el nuevo diseño representa un balance temporal entre las características del producto $\mathrm{y}$ el requerimiento del usuario. Al establecer este feedback se introduce una nueva definición denominada como re-innovación., por ejemplo, realizar un rediseño del producto en función de la satisfacción del consumidor, es asumido como un cambio tecnológico para continuar incrementando las actividades de investigación y desarrollo.

Igualmente, Rothwell afirma que el éxito de la innovación tecnológica en las organizaciones radica en el fortalecimiento del enlace de los productores con el usuario, ya que con esto se lograría la identificación más adecuada para la interpretación de las especificaciones del usuario en el diseño del producto. Rothwell (1986), también afirmó que los usuarios tienen un importante rol en el proceso de la reinnovación, pasan de un rol pasivo a un rol mucho más activo, considerándolos, incluso, como la principal fuente del know-how básico tecnológico al hacerse efectiva la retroalimentación en el sistema.

Posteriormente, diversos inves- tigadores, entre ellos economistas, sociólogos, entre otros, vieron la necesidad de ahondar en el desarrollo de las organizaciones, especialmente, Drucker (1985), considerado por muchos como el padre de la administración de empresas, relaciona en su trabajo Innovation and Entrepreneurshiplos conceptos de innovación y capacidad emprendedora, dando por sentado que la innovación sistemática consiste en una búsqueda organizada con un objetivo de cambios, siendo la herramienta necesaria paraaprovechar la oportunidad de un negocio o servicio diferente.

a partir de lo anterior, Drucker (1985) introduce el concepto de gestión tecnológica, caracterizando a un empresario innovador como quien contribuye a hacer productivas las ideas hasta convertirlas en innovaciones. Adicionalmente, Druker en 1986 propugna la innovación en todos los campos, no solo en el alto desempeño de la tecnología, sino que menciona dos nuevas orientaciones a considerar como parte del estudio, las cuales son el planteamiento de la solución del desempleo y la reducción de un número considerable de instituciones y políticas públicas que según sus apartados los catalogaba como desactualizadas e ineficientes. (Murillo, 2005).

Aunado a las anteriores investigaciones, se le suma lo expuesto por Porter (1990) cuando señala sobre el rol del cambio tecnológico en la alteración de la estructura de la industria para toda empresa que considere la innovación, al respecto, refiere que la innovación aumenta la ventaja competitiva de las compañías,las empresas deben reconocer el rol dual del cambio tecnológico en la formación de una ventaja competitiva y la estructura 
Gestión de Innovación tecnológica y globalización como factores impulsadores de la calidad de servicio y competitividad

industrial cuando selecciona la estrategia tecnológica adecuada juntamente con la inversión tecnológica (Porter,1990).

Por su parte, Ercorsa y Pasola (1997), afirman que la innovación puede abarcar aspectos tanto económicos como tecnológicos, basando su corriente de pensamiento en los escritos de Schumpeter, y tomando como premisa que la innovación está comprendida desde la introducción al mercado de un nuevo producto hasta la conquista de una nueva fuente de materias primas.

Posteriormente, surgen otras investigaciones en el campo de la gestión de innovación tecnológica, resaltando los trabajos de Hamel (2000), Betz (2010), quienes acuerdan que el proceso de innovación es complejo e involucra desde su generación un compromiso de la empresa con la sociedad. El desarrollo de nuevas ideas y la participación de la demanda en el análisis económico de la inversión de tecnologías novedosas hace que cada día exista un motivo más para el avance en materia de competencia tecnológica en las organizaciones de manera global.

\section{Gestión de la innovación tecnológica y calidad de servicio}

El reto inicial de todo gerente en la cadena corporativa es mejorar la eficiencia y crear una competencia sostenible en la organización. De acuerdo con Betz (2011), la innovación tecnológica desempeña un papel protagónico en las empresas ayudando a los gerentes a alcanzar sus objetivos; asimismo, afirma, todos los elementos y actividades pueden desarrollarse efectivamente en conjunto con una estrategia de innovación, permitiendo la generación y optimización de las ganancias, crecimiento continuo, bienes y servicios de calidad; así como, remuneraciones justas para los empleados.

En importante mencionar que la gestión de innovación tecnológica reúne una serie de elementos vitales para cumplir los objetivos definidos en el proceso de planificación estratégica: estrategia de innovación, redes y comunidades de la innovación, investigación y desarrollo, diseño de un nuevo producto o servicio, operaciones y entregas (Dodgson, 2008).

La innovación tecnológica en las organizaciones involucra más que una aplicación exitosa de nuevas ideas de bienes o servicios, muchas veces requiere de cambios organizacionales y estrategias que lo soporte, por ello, la innovación estratégica está dentro del dominio del pensamiento estratégico orientado a la toma de decisiones en el presente para visionar y guiar el futuro de la organización.

Vale la pena destacar que la innovación tecnológica tiene mejores resultados cuando es gestionada de forma conjunta, es decir, varias organizaciones que trabajan de forma mancomunada dentro de la cadena de valor, por ejemplo, proveedores y distribuidores, trabajando en forma de redes o comunidades dando lugar a las colaboraciones tecnológicas.

La gestión de innovación tecnológica para la creación de los productos (bienes o servicios)incluye diversos factores de eficiencia y eficacia, donde el nuevo diseño de un bien o su modificación define de una forma novedosa cómo beneficiará al cliente final de acuerdo con las necesidades del mercado o, simplemente, creando tales oportunidades según la estrategia 
corporativa. La política de atención al cliente que impacta los servicios, sean estos apoyo a la venta de los bienes o la razón de ser de la organización, por ejemplo, un banco, una institución de salud, entre otras, es fundamental para alcanzar la calidad.

Adicionalmente, otro factor importante a considerar es que actualmente las organizaciones se desenvuelven en un ambiente donde se hace más difícil competir. El devenir de los años en estas últimas décadas ha generado un crecimiento mucho más vertiginoso en la tecnología de procesos y productos que antes. La llave que busca toda organización para lograr ser competitivo en el mercado está en lo que dice la frase célebre de Bill Gates el famoso empresario informático cofundador de la empresa Microsoft junto con Paul Allen: "La clave del éxito en los negocios está en detectar hacia dónde va el mundo y llegar ahí primero", evidentemente, fue un hombre visionario que supo identificar con mucha antelación la necesidad que se crearía en el mundo de la informática por lo que decidió innovar.

Por otro lado, las empresas de servicios para llevar a cabo la gestión de innovación tecnológica, por la naturaleza de su actividad requieren de un tratamiento especial y no está caracterizado de forma similar con respecto a las empresas que solo se encargan de la venta de productos tangibles, Kotler (2014), define los servicios como una forma de producto cuya intensión consiste en beneficios o satisfacciones ofrecidas a la venta, siendo intangibles y no tienen como resultado la propiedad de algo. Dentro de esta forma de servicios entran en clasificación lo ofrecido en hoteles, bancos, aerolíneas, mantenimiento y reparaciones, entre otras.

Vale destacar, que al mencionar las características de los servicios se aclararía mucho más su naturaleza y diferencia con respecto a los bienes. De acuerdo con Grande (2005) dentro de los aspectos más resaltantes de los servicios se encuentra la intangibilidad que al parecer es la primera dimensión que lo describe y la que marca la distancia entre bienes y servicios de forma notoria.

La intangibilidad se refiere a que los servicios no se pueden apreciar directamente por los sentidos antes de adquirirlo, por lo tanto, no se pueden patentar por lo que no goza el beneficio de propiedad que poseen los bienes, por su exclusividad los servicios no pueden probarse antes de "comprarse" lo que genera dificultades mayores en las barreras de entrada al sector según. Las empresas que se dedican a ofrecer servicios por lo general deben tener una imagen corporativa frente a la competencia y esmerarse en la calidad y reputación ante sus competidores (Grande, 2005).

Asimismo, la inseparabilidad (Martín y Garrida, 2016) es una característica bastante peculiar ya que los servicios no pueden darse sin contacto entre el cliente y el proveedor que es quien lo produce, dándose lugar su creación mientras se consume, siendo fruto del esfuerzo en conjunto del proveedor y el cliente. De hecho, cuando los bienes se producen, los mismos son almacenados, se venden y luego se consumen mientras que los servicios se venden y después se producen, se consumen y no se pueden almacenar.

Otra característica fundamental en los servicios es su carácter de heterogeneidad como resultado de medidas que son personalizadas según 
lo requerido del cliente. Por ser más difícil de estandarizar los servicios ya que los mismos son percibidos en función de las personas que lo ofrecen, cuándo y dónde los ofrecen y tiene sus variantes respectivas dependiendo también de las empresas y qué tipo de cliente lo está necesitando, haciendo este proceso más complejo aún. Por lo tanto, la fijación del precio y el control de calidad según Martín y Díaz (2016) son elementos que requieren de una evaluación más extensa en comparación a la venta de bienes intangibles jugando un papel fundamental la tecnología y sus aplicaciones y usos en la organización.

De forma similar, el carácter perecederodelosservicioslocaracterizan diferenciándolo de los bienes; se dice que un servicio es perecedero cuando no es posible almacenarlos para consumirlos luego ya que la producción y el consumo son inmediatos, en otras palabras, los servicios carecen de inventario; sin embargo, dependiendo de la naturaleza del servicio algunos, en particular pueden almacenar parte de sus procesos pero no podrá ser posible inventariar por decirlo de una manera $u$ otra la experiencia del cliente.

Ahora bien, de acuerdo con Gronroos (1995) para garantizar un servicio excelente y que cubra las expectativas del cliente y den un valor agregado adicional es importante que el mismo se ofrezca con un alto nivel de calidad. Este último, es un concepto amplio y complejo en donde los servicios se experimentan de forma subjetiva y las actividades de producción y consumo se realizan de forma simultánea. De esta manera la calidad del servicio, debe ser tal cual como es percibida por el cliente, y la misma tiene dos dimensiones, una técnica o de resultado y una funcional relacionada al proceso, nuevamente se evidencia como el uso de la tecnología puede impactar los resultados y la percepción de los clientes (Gronroos, 1995).

La dimensión técnica está referida al conjunto de esfuerzos que se llevan a cabo para que el producto ofrecido continúe cumpliendo con las especificaciones a la cual fue diseñado aportando un valor agregado a la actividad, mientras que la dimensión funcional se refiere más bien a cómo se llevará a cabo el proceso y si fue complicado o sencillo asumirlo por el usuario; por ejemplo cuando una queja del cliente es resuelta de forma tardía, la calidad funcional del proceso como tal se deteriora por una mala imagen adquirida por el cliente, en todo momento los procesos deben estar diseñados para dar respuestas oportunas y acordes con el servicio, el uso de la tecnología para tal fin, permite la automatización y la sincronización entre departamentos y funciones.

Es así que, además de la calidad en el servicio aportada al cliente, autores como Kotler (2014), consideran también de gran importancia en la actualidad del marketing las experiencias, ya que éstas siempre han formado parte importante en la comercialización de productos de algunas compañías marcando una gran diferencia con respecto a aquellas que no lo toman en cuenta. Muchas empresas están pasando a un nuevo nivel de creación de nuevo valor para sus clientes de manera tal que el producto ofrecido debe garantizar dejar en la mente del cliente la satisfacción de disfrutar y cubrir sus necesidades trayendo como consecuencia la continuidad y preferencia en la solicitud por parte del interesado.

Según artículo publicado por la revista Business Week (1999), los 
cambios que experimenta el mercado están impulsados por lo que el consumidor aspira, es decir, una combinación adecuada de calidad del bien, con el precio correcto y un buen servicio, es importante recordar, la entrega un no es suficiente, esto lleva intrínseco un valor agregado, generalmente relacionado con el servicio, las actividades posventa, entre otros atributos, lo que genera la creación de lealtad del cliente.

Un ejemplo mencionado por Vilagines (1999) refiere una entrevista realizada al señor Michael Dell acerca de su compañía en la cual expresó las razones por las cuales logró un gran crecimiento y una gran ventaja competitiva; su éxito está basado en el marketing directo, configuración adaptada al cliente y apoyo (servicio) directo al cliente. Para Dell, las llamadas del cliente son primordiales a la hora de la toma de decisiones, por lo que la política se basa en traspasar de forma inmediata los casos críticos al Dpto. de investigación y desarrollo, permitiendo hacer cualquier ajuste en las especificaciones técnicas del producto o simplemente modificando cualquier aspecto de la calidad.

En contraposición a la experiencia de Dell, diversos autores como Vértice (2000) afirman que dependiendo del estatus del ciclo de vida del producto, si están ubicado en la fase de madurez llega un momento en el cual un determinado bien se consolida en el mercado y no es necesario una inversión adicional ya que "se venden solos" siendo un producto muy conocido y con mucha clientela que se ha logrado precisamente por la consolidación en el mercado a través de estrategias de marketing diferenciadoras.

Después de lo anteriormente expuesto acerca del abordaje de la gestión de innovación tecnológica en organizaciones dentro del área de servicios es importante conocer sus inicios, los factores y las causas que se fueron desencadenando para dar lugar a la lucha por la competitividad en el mundo tan cambiante del día de hoy a través de la globalización, las fuentes correspondientes de la innovación tecnológica y las actividades que se desarrollan en los departamentos o áreas dedicadas a la investigación y desarrollo.

\section{Globalización: factor impulsor en la gestión de innovación tecnológica.}

La globalización es un fenómeno que ha impactado y cambiado la forma de hacer las cosas, espacios personales, sociales, profesionales y laborales se han visto afectados por los efectos de la globalización resultada de las tecnologías de información y comunicación, una innovación que sin lugar a dudas a cambiado el mundo. La globalización trajo consigo procesos radicales, nuevas necesidades y generación de expectativas, cambios en los cultural, transcendencia de lo nacional a lo internacional, más aun, de los regional a lo mundial.

La necesidad de lograr diferentes intercambios económicos, políticos, sociales y culturales, generó nuevas formas organizativas para adaptar los negocios, el comercio y la producción, como resultado surgen países altamente industrializados que lideran el mercado del libre comercio y que requieren necesariamente de una integración para su dinamismo en el ámbito local, nacional e internacional. Bravo (2006) apoya la idea de que el inicio de la 
globalización se dio desde el comienzo del modelo capitalista como sistema económico, seguido por la revolución industrial con el perfeccionamiento de la máquina de vapor, la maquinaria textil y de producción de acero.

Evolutivamente, desde la mitad del siglo XIX, surge la invención del ferrocarril y el telégrafo, seguidamente, la electricidad, recurso energético que es sinónimo de desarrollo, por mencionar solo algunas de las invenciones que han transformado las sociedades y por ende las naciones. De esta manera se puede indicar que la necesidad de cubrir el mercado por las crecientes tensiones económicas, de alguna u otra manera cooperó en la creación de nuevas inventivas en varios sectores de la economía, incluyendo el tecnológico.

Otro factor que ha cooperado con el desarrollo de la globalización es la especialización y la división del trabajo técnico a nivel mundial según lo aportado por Capdevielle (2000) quien expresa que el carácter heterogéneo y especializado de las economías regionales denota actividades productivas con niveles desiguales de productividad y en consecuencia de remuneraciones, por tal motivo, resulta imperante analizar la innovación tecnológica como factor competitivo dentro de las organizaciones y que en conjunto con la globalización ha promovido la competitividad y la consecuente mejora de los bienes y servicios.

Según, Escorsa y Valls (2003), los productos se hacen más competitivos mediante las innovaciones. La Cámara Oficial de Comercio e Industria de Madrid (2004) conceptualiza la innovación como el proceso de conversión de ideas en productos o servicios nuevos o mejorados que aporten rendimiento al mercado y beneficios a la empresa objeto del proceso innovador. De forma similar, la innovación es definida de acuerdo con el Manual de Frascati de la Organización para la Cooperación y el Desarrollo Económicos conocido en sus siglas como OCDE como la transformación de un producto, idea o servicio que sea comercializable, $\mathrm{O}$ un procedimiento de fabricación o distribución nuevo o mejorado, incluso, la transformación a un nuevo método para proporcionar un servicio social.

Por otro lado, debido a que el ciclo de vida de los productos se hace cada vez más corto (Figura 1), la presión aumenta entre las organizaciones para alcanzar una ventaja competitiva. Escorsa y Valls (2003) señalan que el progreso técnico, la internacionalización de la economía y la desmasificación de los mercados son tendencias que generan un mayor número de productos en el mercado. Se hacen productos más personalizados en función de solventar la necesidad del mercado que se agudiza más debido a la competencia por la permanencia.

En consecuencia, Mandado et al, (2003), señalan las fases constituyentes del ciclo tecnológico, consecuencia de la globalización, tal como: iniciación, explosión, madurez y declive. La primera fase denominada iniciación, es el periodo de aparición de una técnica que se origina por una invención reciente. Las ventas en esta fase comienzan a crecer lentamente siendo menor su rendimiento. Existen dificultades ya que posiblemente existan deficiencias tecnológicas que deban ser aclaradas.

La segunda etapa es la explosión, constituye una mejora en la técnica y es más confiable que en el primer ciclo. El producto en esta etapa va incrementando su aceptación con el aumento de las ventas, a su vez pueden existir cambios 


\section{Figura 1 \\ Curva en S del ciclo de vida tecnológico.}

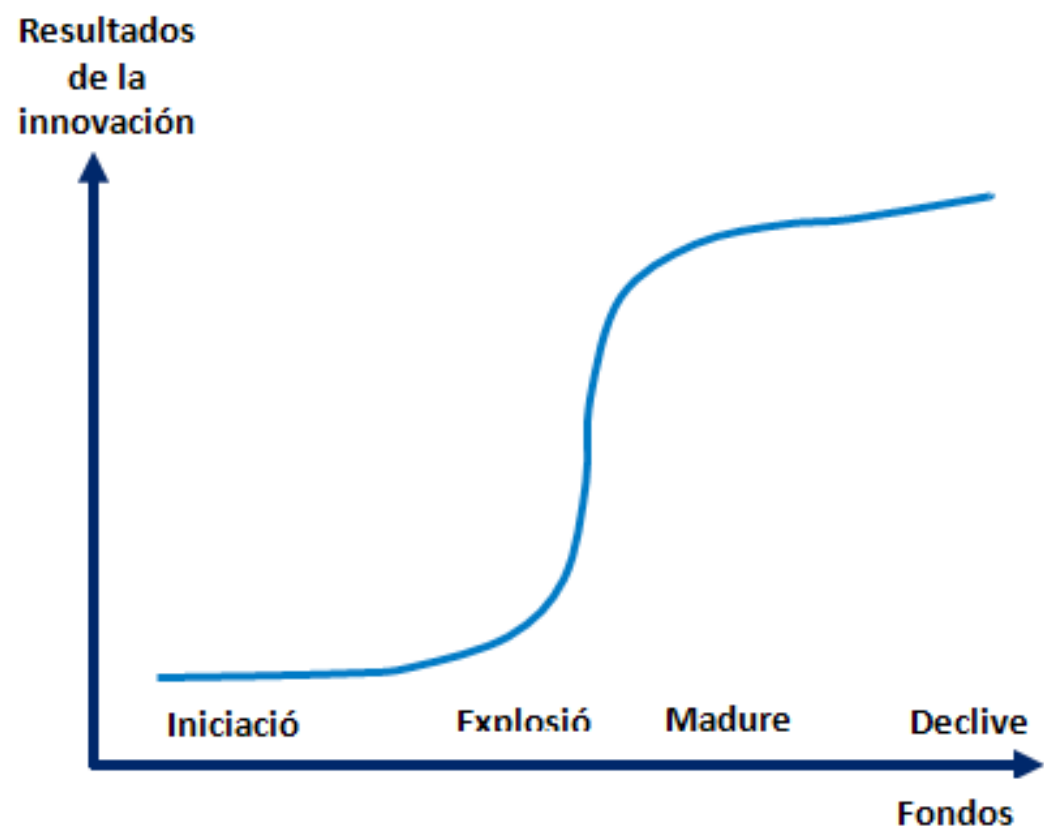

Fuente: Mandado et al, (2003)

como consecuencia de su aplicación y aceptación, surgen nuevas expectativas de ventas. Luego de esta etapa entra en juego la madurez, ésta constituye una fase en la cual la técnica aplicada se estabiliza en procedimientos y experiencia, además de considerar que el producto es vendido en grandes cantidades y las competencias se basan en los precios y la diferenciación.

Posteriormente a las etapas mencionadas, se encuentra la última que cual está categorizada como el punto de saturación o declive donde disminuyen los compradores y donde el avance de la técnica llega a su límite; la misma se presenta cuando las ventas disminuyen significativamente y probablemente otras tecnologías más novedosas han sustituido al producto, o tienen más aceptación en el mercado.

Para el caso de las grandes empresas de servicios integrales, uno de los factores de la gestión que más influyen para su progreso es la innovación tecnológica de los productos ofrecidos al mercado. Principalmente la tecnología es un término acuñado desde inicios del siglo XIX, poco a poco, gracias a su evolución ha permitido el incremento de diversas técnicas mediante el conocimiento exacto de los principios físicos. (Mandado et al., 2003). Al respecto, Mandado et al. 
(2003), afirman que la tecnología siempre ha modificado de alguna manera la naturaleza y puede destruir su equilibrio, la fabricación de productos siempre generan desechos, para lo cual se han establecido ciertas regulaciones en este ámbito, $\mathrm{m}\{$ as allá, la innovación tecnológica, no tendrá valor si no se aplica para resolver un problema práctico, de allí, los programas de investigación y desarrollo, así como, la gran importancia del quehacer científico tecnológico en las empresas en general.

Precisamente, el conocimiento científico tecnológico ha evolucionado para lograr adaptarse a los fuertes cambios resultado de la globalización, cada vez más se hace necesario empresas comprometidas con el desarrollo, motivadas por la mejora de la competitividad y por el crecimiento de un sector y de la economía en general, la globalización hace indispensables los procesos de innovación para generar ventajas competitivas en los mercados; pero, sobre todo, para producir soluciones a los problemas de la sociedad,

Por lo tanto, el término de innovación tecnológica cobra valor en la actualidad y es definida por Hidalgo y Pavón (2002) como un proceso que incluye la invención y explotación técnica y comercial de productos o servicios cuyas actividades están dirigidas a la generación de nuevas ideas y su implementación. El proceso de explotación en sí quiere decir la transformación de esas ideas en productos, procesos técnicos y organizativos nuevos 0 mejorados mediante el desarrollo, la fabricación y la comercialización, involucrando por supuesto la transferencia de los resultados obtenidos y su transmisión por medio de la comercialización.

\section{Conclusiones}

El impacto que la globalización trajo al mundo no deja de ser un reto permanente en todos los espacios en donde se desempeña el ser humano. La gestión de la innovación tecnológica no es más que la necesidad que se presenta en las empresas de aprovechar los recursos tecnológicos en pro de los procesos y productos organizacionales.

Las organizaciones tienen que adoptar tecnologías de avanzada de forma innovadora, su uso debe beneficiar al cliente final y debe estar orientado a la optimización de los recursos humanos, económicos y por supuestos tecnológicos. La planificación, ejecución, seguimiento y control en base a las innovaciones tecnológicas representan un gran reto por la imperiosa necesidad de ser visionarios y adelantarse a lo que está por venir.

Sin lugar a dudas, y comparado con otros aspectos de la organización la gestión de innovación tecnológica es un término relativamente reciente que nace por la necesidad de fusionar y adaptar los procesos relacionados con las ciencias administrativas a los avances de la ciencia y la tecnología, generando una empresarial cambiante, en el mejor de los casos, moldeable o adaptable a los retos que se presenten.

En este sentido, se puede concluir que la evolución de la gestión de innovación tecnológica tiene varias aristas y diferentes puntos de vista, muchos autores coinciden y defienden las bondades de sus avances básicamente los que apoyan el capital mientras que los más humanistas cuestionan el uso de las tecnologías por desplazar la mano de obra calificada dejando a un lado la fuerza laboral y el 
detrimento de las condiciones sociales de los trabajadores.

indiscutiblemente, el usos de las tecnologías favorece los procesos organizacionales, su aparición y uso transformó la manera en que se produce y se llevan a cabo los procesos en las empresas, aquellas empresas con mejor gestión de la innovación tecnológica, sin lugar a dudas, obtendrá mejores resultados, no por casualidad, las empresas con mejores resultados económicos, están relacionadas con tecnología.

Así pues, la globalización apoya los procesos de socialización de las innovaciones y juega un papel fundamental en la comercialización de productos cada vez más innovadores y competitivos, consecuentemente, la menor capacidad de innovación o de adopción tecnológica, produce un rezago en el desarrollo de las organizaciones y en la generación de ventajas competitivas, produciendo, a su vez, distorsiones en el crecimiento de las empresas y de los países.

\section{Referencias bibliográficas}

Betz, Frederick (2011), Managing Technological Innovation: Competitive Advantage from Change. Tercera edición. John Wiley and Sons Inc. New Jersey. Canadá,

Bravo, Ernesto (2006), Globalización, innovación tecnológica y pobreza. Aproximación a las nueve conceptualizaciones en Latinoamérica. Espacio Abierto, Vol. 21, Núm. 3, julio-septiembre, 2012, pp. 543-556 Universidad del Zulia, Venezuela

Cámara Oficial de Comercio e Industria de Madrid (2004), Herramientas de Gestión de la Innovación. Legna Gráficas, S.L. pp. 10.
Dodgson, Mark; Gann, David Gann; Salter, Ammon (2008), The Management of Technological Innovation: Strategy and Pratice. OUP Oxford.

Drucker (1986), Innovation and entrepreneurship: practice and principles,

Drucker Peter (1985), Innovation and Entrepreneurship by Peter F. Drucker, https://doi.org/10.1002/ npr.4040050112

Escorsa Castells, Pere y Valls Pasola, Jaume (2003), Tecnología e innovación en la empresa. Primera Edición Ediciones UPC. Barcelona, España.

Escorsa Pere; Pasola Jaume (1997), Manual de gestión e innovación tecnológica en la empresa, Editorial Santiago de Chile: CINDA.

Fernández Romero, Andrés (2005), Creatividad innovación en empresas y organizaciones: Técnicas para la resolución de problemas. Ediciones Díaz de Santos, S.A. Madrid España.

Freeman Christopher (1982), The Economics of Industrial Innovation, Thhttps://ssrn.com/abstract $=1496190$

Gary Armstrong, Stewart Adam; Sara Denize; Philip Kotler (2014), Principles of Marketing, edición Pearson Australia, 20141486002536, 9781486002535

Grande Esteban, Ildefonso (2005),Marketing de los Servicios. 4ta Edición. Esic Editorial. Madrid, España.

Gronroos, Christian (1995), Marketing y gestión de servicios. la gestión de los momentos de la verdad y la competencia en los servicios. Ediciones Díaz de Santos. Madrid España

Hamel, Gary (2000), Liderando la revolución. Grupo Editorial Normal. Bogotá. 
Gestión de Innovación tecnológica y globalización como factores impulsadores de la calidad de servicio y competitividad

Ana María, Ramos Vera

\section{Colombia.}

Hidalgo, Antonio; León, Gonzalo; Pavón, Julián (2002), La gestión de la innovación y la tecnología en las organizaciones. Pirámide, Madrid.

Interempresas (2017), Las tendencias tecnológicas para 2018. Artículo digital. Visitado el 22-12-2017: https://www.interempresas.net/Robotica/Articulos/205939-Las-tendencias-tecnologicas-para-2018.html.

Malthus, Thomas (1973), Principios de Economía Política. Fondo de Cultura Económica. México.

Mandado, Enrique; Fernández, Francisco (2003), La innovación tecnológica en las organizaciones. Thompson editores, España.

Martín Peña, María Luz y Díaz Garrida, Eloisa (2016),Fundamentos de dirección de operaciones en empresas de servicios. Esic Editorial. Madrid, España.

Marx, Karl (1978), El capital. Crítica de la economía política. Vol. I. (2 ${ }^{a}$ Edición. $113^{a}$ Reimpresión). Fondo de Cultura Económica. México.

Murillo Javier (2005), La Investigación sobre Eficacia Escolar. Barcelona:
Octaedro, Universidad Autónoma De Madrid, España.

Organización para la Cooperación y el Desarrollo Económicos (2002), Frascati Manual: Proposed standart practice for survays on research and experimental development.

Porter, Michel (1990), The Competitive Advantage of Nations, Nueva York.

Ricardo, David (1973),Principios de Economía Política y Tributación. Fondo de Cultura Económica.México.

Schumpeter Joseph (1942), Capitalismo, Socialismo y Democracia.3ra. Edición. London: George Allen and Unwin, 1974

Smith, Adam (1996),Investigación sobre la naturaleza y causas de la riqueza de las naciones. (Reproducción facsímil de la primera edición en castellano de publicada en Valladolid en 1794). Junta de Castilla y león. Valladolic. España. Vol I. página 12.

Veblen, Thorstein (1965), The engineers and the price system. August M. Kelley. Mew Jersey.http:// stolpkin.net/spip.php?article696

- Esta obra está bajo una licencia de Creative Commons Reconocimiento-NoComercial-

Compartirlgual 3.0 Unported.

http://creativecommons.org/licenses/by-nc-sa/3.0/deed.es_ES 